\title{
Rainbow Phenomena in Particle Scatterings
}

\author{
Ju-Tang Hsiao ${ }^{1}$ and Keh-Ning Huang ${ }^{1,2}$ \\ ${ }^{1}$ Department of Physics, National Taiwan University \\ Taipei, Taiwan 106, Republic of China \\ ${ }^{2}$ Institute of Atomic and Molecular Sciences, Academia Sinica \\ P.O. Box 23-166 Taipei, Taiwan 106, Republic of China
}

\begin{abstract}
The Scattering of a particle in quantum mechanics and the scattering of electromagnetic waves have certain analogies. The quantum scattering of a particle is analyzed as the interference among components characterized by number of reflections at the spherical boundary in analogy to the rainbow phenomena of light.
\end{abstract}

The scattering of a particle in quantum mechanics and the scattering of electromagnetic waves have certain analogies. The differential cross section of the scattering of a particle with mass $m$ and energy $E$ by a spherical square-well potential of the form

$$
V(r)=\left\{\begin{array}{cc}
0 & , r>a \\
-V_{0} & , r \leq a
\end{array}\right.
$$

is analyzed as the interference among components characterized by number of reflections at the spherical boundary in analogy to the rainbow phenomena of light. Let 1 and 2 denote, respectively, the exterior and interior regions of the sphere. The differential cross section can be written as

$$
\frac{d \sigma}{d \Omega}=\frac{1}{4 k^{2}}\left|\sum_{l=0}^{\infty}(2 l+1) P_{l}(\cos \theta)\left\{R_{1}-1+T_{1} T_{2} \sum_{N=0}^{\infty} R_{2}^{N}\right\}\right|^{2} \quad, k=\sqrt{2 m E} / \hbar
$$

where $R_{1}$ and $T_{1}$ are the external reflection and transmission coefficients from region 1 to 2 , while $R_{2}$ and $T_{2}$ are the internal reflection and transmission coefficients from region 2 to 1 . The first term $R_{1}$ inside the curly brackets represents the direct external reflection from the potential well. The $N$ th term in the second summation corresponds to the component that transmits into the potential well with $N$ internal reflections and transmits outside finally. 